\title{
Improvement of the spatial beam quality of laser sources with an intracavity Bragg grating
}

\author{
Sylvie Yiou, François Balembois, and Patrick Georges \\ Laboratoire Charles Fabry de l'Institut d'Optique, Unité Mixte de Recherche 8501 du Centre National de la Recherche Scientifique, \\ Centre Universitaire, 91403 Orsay Cedex, France \\ Jean-Pierre Huignard \\ Thalès Research and Technology France, Domaine de Corbeville, 91404 Orsay Cedex, France
}

Received July 8, 2002

\begin{abstract}
We demonstrate a novel method for improvement of spatial beam quality by use of the angular selectivity of a thick phase grating inserted into various laser cavities. Preliminary experiments with an intentionally degraded beam from a Nd: $\mathrm{YVO}_{4}$ laser have enhanced beam propagation factor $M^{2}$ by a factor of 4.5. Operation with a broad-area laser diode in an external cavity with a thick grating was achieved. We obtained $100 \mathrm{~mW}$ of cw power in a beam with an $M^{2}$ of 1.3. Moreover, a reduction of the output spectrum by as much as a factor of 3.8 was obtained. (C) 2003 Optical Society of America

OCIS codes: $140.5960,050.7330$.
\end{abstract}

Conventional high-power diode lasers exhibit poor spatial beam quality along their slow axes because they are 10-50 times more divergent than the limit of diffraction in that direction. These typical values depend on the type of diode (single element or array) as well as on its output power. This major drawback makes such diodes unsuitable for many industrial applications or for the pumping of erbium-doped fiber amplifiers for telecommunications. Many approaches to overcome this limitation have been proposed. On the one hand, monolithic integrated devices have been demonstrated in the laboratory to yield powers of a few watts in a transverse single mode. ${ }^{1,2}$ However, their specific technologies are not yet perfectly controlled. On the other hand, it is interesting to use off-the-shelf laser diodes that have been developed for pumping different types of laser gain media. In particular, the use of external cavities remains of great interest because of the compactness and simplicity afforded by these cavities compared with injection locking by a separate master laser. ${ }^{3}$ Various kinds of cavities have been studied, among them an external cavity that uses a butt-coupled graded-index lens and a striped gold mirror, ${ }^{4}$ an external cavity with an external mirror in the image plane of the junction, ${ }^{5}$ off-center external cavities, ${ }^{6}$ and cavities that use photorefractive phase conjugation. ${ }^{7,8}$ More recently, $\mathrm{TEM}_{00}$ operation yielding $400 \mathrm{~mW}$ of $\mathrm{cw}$ power was achieved in an external cavity with two crossed cylindrical resonators. ${ }^{9}$ In this Letter we demonstrate a new external-cavity technique that allows for efficient spatial mode selectivity. The idea is to use the angular selectivity of a thick Bragg grating to achieve spatial filtering in an external cavity. To validate the principle of spatial filtering by an intracavity thick grating, we carried out preliminary experiments with an intentionally degraded beam from a diode-pumped $\mathrm{Nd}: \mathrm{YVO}_{4}$ laser. First we present the results obtained with this configuration. Then we describe the results obtained with two broad-area semiconductor laser diodes.
The key component in achieving mode filtering is a thick transmission-type phase volume grating, which may exhibit both high diffraction efficiency and a sharp angular bandwidth near Bragg incidence. From the various holographic photopolymer materials we chose to use phenanthrenquinone-doped poly (methyl methacrylate), which can be as thick as several millimeters with good optical quality. ${ }^{10,11}$ Because Bragg selectivity $\Delta \theta$ of an elementary Bragg grating is of the order of $\Delta \theta=n p / e$ ( $n$ is the refractive index; $p$ is the grating period; $e$ is the material thickness), we achieved an angular bandpass of a few milliradians with the following conditions: $p=7 \mu \mathrm{m}$ and $e=2.4 \mathrm{~mm}$. The grating was recorded with an argon laser at $488 \mathrm{~nm}$. Its intrinsic Bragg diffraction efficiency was 85\% at 971 and $1064 \mathrm{~nm}$, and the full width at half-maximum of the angular selectivity curve was $3.5 \mathrm{mrad}$.

Preliminary experiments were carried out at $1064 \mathrm{~nm}$ with a beam that was intentionally degraded in the lateral direction and diffraction limited in the other direction [Fig. 1(a)]. We obtained this beam from a $\mathrm{Nd}: \mathrm{YVO}_{4}$ laser by using a diode pump beam in the crystal that was three times larger than the fundamental mode of the cavity in the lateral direction. The diffraction limit of the $\mathrm{TEM}_{00}$ laser beam in the output arm was approximately $2.9 \mathrm{mrad}$. The degraded laser had a beam propagation factor $M^{2}$ of 4.5. The maximum output power was $325 \mathrm{~mW}$ for an absorbed pump power of $1.52 \mathrm{~W}$, and the laser reached threshold for an absorbed pump power of $0.35 \mathrm{~W}$, leading to a slope efficiency of $28 \%$. Without any change in the pump optics, we inserted the grating into the laser cavity [Fig. 1(b)]. A highly reflecting (HR) mirror coupled the diffracted beam back into the crystal to force the laser to oscillate on its fundamental mode. The main output beam was the beam that was directly transmitted by the grating at the first pass [the second pass in the grating resulted in a second output beam, representing $\sim 10 \%$ of the total output 


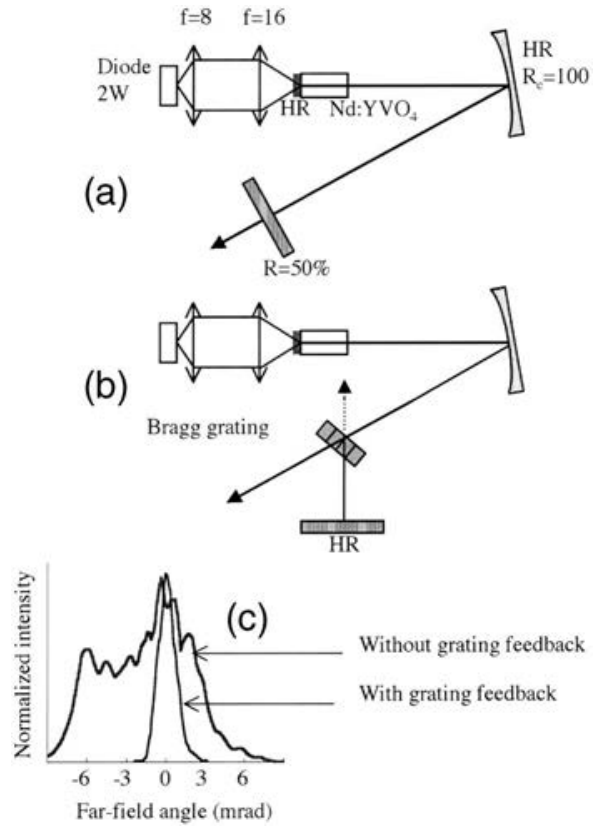

Fig. 1. (a) Laser cavity providing a degraded beam in the lateral direction (without Bragg grating). (b) Laser cavity operating with grating feedback. (c) Far-field patterns produced by the two cavities. Distances are in millimeters.

power; see Fig. 1(b)]. $M^{2}$ was reduced to nearly 1.0. The maximum total output power was $195 \mathrm{~mW}$ for an absorbed pump power of $1.52 \mathrm{~W}$, and the laser reached threshold for an absorbed pump power of $0.66 \mathrm{~W}$. Then the slope efficiency was reduced only to $23 \%$. Figure 1(c) illustrates the improvement in beam quality.

These results have encouraged us to apply this technique to a diode laser in an external cavity. Both laser diodes employed were broad-area laser diodes with an emitting surface of $1 \mu \mathrm{m} \times 100 \mu \mathrm{m}$ and an emission wavelength near $970 \mathrm{~nm}$. The first diode $(970 \mathrm{~nm}, 2 \mathrm{~W}$, from Sacher Lasertechnik $\mathrm{GmbH}$ ) had an antireflection coating on the outcoupling facet $(R=0.1 \%)$ that raised the laser threshold from 540 to $600 \mathrm{~mA}$. Figure 2 shows the experimental setup. Among the various external cavity configurations that have been proposed and analyzed, ${ }^{4-9}$ we preferred to choose one that uses cylindrical lenses, as our component operated in one dimension. The fast axis of the diode is collimated by an aspherical fast-axis collimator (FAC) of 0.91-mm focal length with a N.A. of 0.85 and an antireflection (AR) coating from 780 to $1050 \mathrm{~nm}$. The angular selection is made by the grating in the slow-axis direction. The external cavity, consisting of a $100-\mathrm{mm}$ focal-length cylindrical lens and a highly reflecting mirror, is a stable resonator in the slow-axis direction. Inasmuch as injection efficiency is optimized for an injection spot size of approximately one half of the width of the device, ${ }^{12}$ the lengths in the feedback arm were adapted to have a beam waist at $1 / e^{2}$ inside the diode of $45 \mu \mathrm{m}$. We could simultaneously display both near- and far-field patterns as well as the spectrum of the output beam.

The use of the grating in our external cavity allowed us to reduce the laser threshold of the free- running AR-coated diode from $600 \mathrm{~mA}$ to $I_{\text {th }}=$ $540 \mathrm{~mA}$. The $100-\mu \mathrm{m}$-wide aperture of the diode corresponds to a diffraction limit of approximately $6.2 \mathrm{mrad}$ for a Gaussian beam. The far-field pattern of the free-running AR-coated diode is represented in Fig. 3 for a driving current of $840 \mathrm{~mA}\left(1.55 I_{\text {th }}\right)$. $M^{2}=8.2$. We observed two different kinds of behavior of the far-field pattern in the external cavity. From 540 to $840 \mathrm{~mA}$ the far-field pattern was dominated by the effect of the grating, and the main lobe of the beam contained almost all the emission. Above $840 \mathrm{~mA}$, the energy was uniformly distributed over $50 \mathrm{mrad}$, and there was a competition between the effects of the grating and of the free-running diode. Best results were obtained at $840 \mathrm{~mA}$ with an output power of $100 \mathrm{~mW}$ in a single lobe containing more than $70 \%$ of the total emission. $M^{2}=1.3$, which corresponds to an improvement in beam quality by a factor of 6 (Fig. 3). The divergence angle at $1 / e^{2}$ was reduced from $50 \mathrm{mrad}$ at $1.5 I_{\text {th }}$ to $8 \mathrm{mrad}$. In Fig. 3 the hole at $5 \mathrm{mrad}$, which is symmetrical to the emitted lobe, corresponds to the direction of the Bragg incident radiation (15\% of which was transmitted by the grating and $85 \%$ of which was diffracted). Moreover, we assume that the local maximum at 11 mrad was due to the secondary peak of the selectivity curve of the grating. Figure 4 represents the cw optical power-versus-current curve with and without the external cavity. The output power of the external cavity was limited because the grating was not AR coated, a condition that affected both injection efficiency and the intensity of the output beam that passes through the grating (the losses that were due

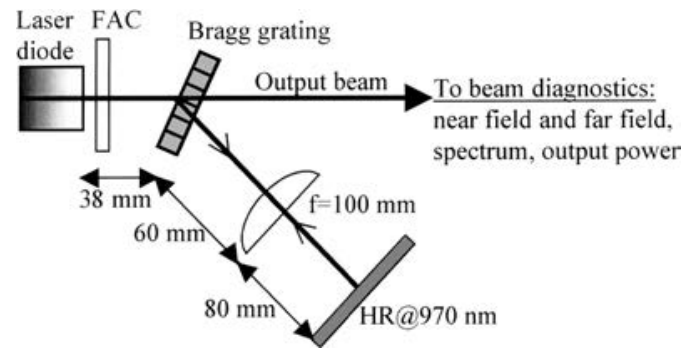

Fig. 2. Experimental setup of the semiconductor laser in an external cavity.

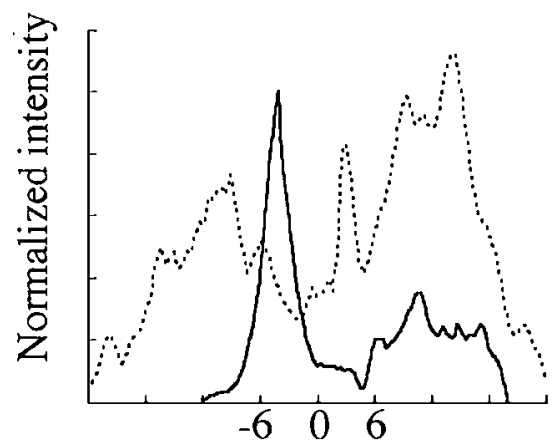

\section{Far-field angle (mrad)}

Fig. 3. Far-field patterns of the AR-coated diode with (solid curve) and without (dotted curve) grating feedback at $840 \mathrm{~mA}$. 


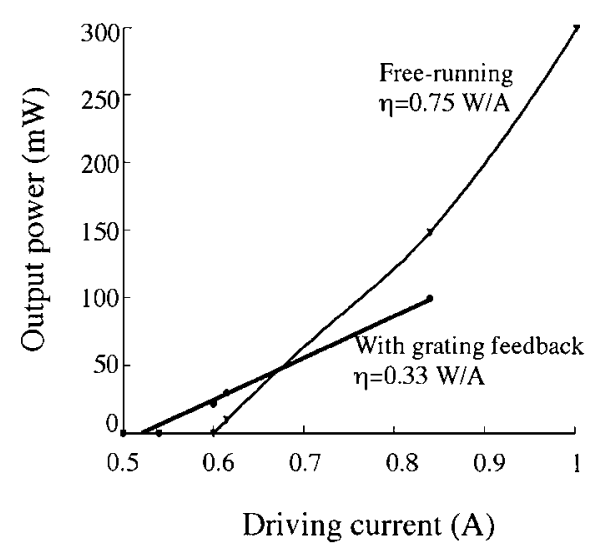

Fig. 4. Output power versus driving current of the AR-coated laser diode with and without grating feedback.

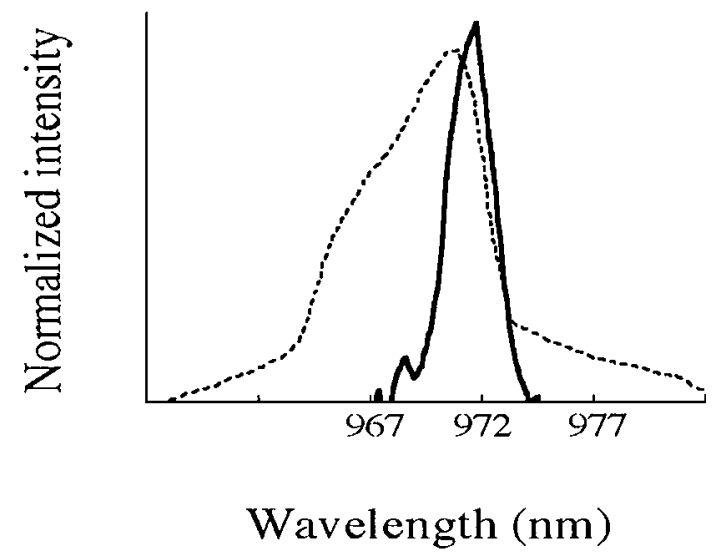

Fig. 5. Output spectrum of the standard diode with (solid curve) and without (dotted curve) grating feedback.

to the grating were $15 \%$ per pass). Moreover, the $100-\mathrm{mm}$ focal-length cylindrical lens was responsible for $7 \%$ of losses per pass.

The second diode was a standard diode with a coating of approximately $R=4 \%$ on the outcoupling facet and a laser threshold at $290 \mathrm{~mA}(4 \mathrm{~W}, 970 \mathrm{~nm}$, from SDL, Inc.). In the external cavity with the grating the laser threshold was reduced to $I_{\mathrm{th}}{ }^{\prime}=280 \mathrm{~mA}$. The far-field pattern was single lobed under a driving current of $360 \mathrm{~mA}\left(1.3 I_{\mathrm{th}}{ }^{\prime}\right)$, and we obtained $220 \mathrm{~mW}$ of power $\mathrm{cw}$ in the main lobe of the far-field pattern with a driving current of $1.5 \mathrm{~A}\left(5 I_{\mathrm{th}}{ }^{\prime}\right)$. That lobe was 2.5 times diffraction limited.

Our external cavity also had an effect on the output spectrum. The spectra of the two diodes were reduced in the external cavity, and the most significant reduction was obtained for the standard diode with a spectral bandwidth reduction of a factor of 3.8 at $I=1.3 I_{\mathrm{th}}{ }^{\prime}$ (Fig. 5).

In conclusion, we have successfully demonstrated a novel compact technique that allows for significant improvement of the beam quality of laser sources. The key component for efficient mode filtering is the phenanthrenquinone-doped poly(methyl methacrylate) transmission-type volume Bragg grating. The first step in the validation of the principle of spatial filtering by an intracavity Bragg grating was achieved with a degraded $\mathrm{Nd}: \mathrm{YVO}_{4}$ laser because nearly $\mathrm{TEM}_{00}$ operation was obtained. Then we applied this technique to an AR-coated laser diode and a standard laser diode. In comparison with external resonators that use nonlinear photorefractive conjugators, ${ }^{7,8}$ our device has a more compact structure: a single Bragg polymer grating provides both spatial and spectral filtering of the modes. The same scheme may apply to a single element or to a diode laser array. Reduction of $M^{2}$ by as much as a factor of 6 was obtained. In addition, a reduction of the optical spectrum of the laser diodes by as much as a factor of 3.8 was observed. After this proof of concept, better results in terms of output power are expected with AR coating of all the components of the cavity and a smaller residual reflectivity of the diode laser's output facet.

We thank Ken Y. Hsu and his colleagues at the Institute of Electro-Optical Engineering, National Chiao Tung University, Hsin-Chu, Taiwan, for providing the phenanthrenquinone-doped poly(methyl methacrylate) samples used in our experiments. S. Yiou's e-mail address is sylvie.yiou@iota.u-psud.fr.

\section{References}

1. S. D. DeMars, K. M. Dzurko, R. J. Lang, D. F. Welch, D. R. Scifres, and A. Hardy, in Conference on Lasers and Electro-Optics, Vol. 9 of 1996 OSA Technical Digest Series (Optical Society of America, Washington, D.C., 1996), p. 77.

2. D. Mehuys, D. F. Welch, R. Parke, R. G. Waarts, A. Hardy, and D. Scifres, Electron. Lett. 27, 492 (1991).

3. L. Goldberg and M. K. Chun, Appl. Phys. Lett. 53, 1900 (1988).

4. C. J. Chang-Hasnain, J. Berger, D. R. Scifres, W. Streifer, J. R. Whinnery, and A. Dienes, Appl. Phys. Lett. 50, 1465 (1987).

5. R. Pillai and E. Garmire, IEEE J. Quantum Electron. 32, 996 (1996).

6. A. Barthelemy, F. Louradour, and V. Couderc, Electron. Lett. 28, 2038 (1992).

7. M. Lobel, P. Petersen, and P. Johansen, J. Opt. Soc. Am. B 15, 2000 (1998).

8. S. MacCormack and J. Feinberg, Opt. Lett. 18, 211 (1993).

9. V. Raab and R. Menzel, Opt. Lett. 27, 167 (2002).

10. S. H. Lin, K. Y. Hsu, W. Chen, and W. T. Whang, Opt. Lett. 25, 451 (2000).

11. G. J. Steckman, I. Solomatine, G. Zhou, and D. Psaltis, Opt. Lett. 23, 1310 (1998).

12. G. L. Abbas, S. Yang, V. W. S. Chan, and J. G. Fujimoto, IEEE J. Quantum Electron. 24, 609 (1988). 